\title{
Human adipose tissue protein analyses using capillary western blot technology
}

Jin Lu ${ }^{1,2}$, Carolyn C. Allred² and Michael D. Jensen ${ }^{2}$

\begin{abstract}
A capillary western blot (Wes ${ }^{\circledR}$ ) technology has recently been validated for analyses of cell culture lysate proteins, but whether it is reliable for human tissue proteins is unknown. We compared traditional western blotting to the Wes ${ }^{\circledR}$ capillary western method to quantitate the relative amount of human adipose tissue CD36, the ratio of phosphorylated Erk1/2 (pErk1/2) to total Erk1/2 during insulin clamp or after niacin treatment and the fold increase in $\mathrm{pAkt}^{5473}$ (Akt phosphorylation on Ser473) in response to feeding. The results from these two methods were highly correlated $(r=0.932$ for CD36, $r=0.905$ for pErk1/2:Erk1/2, $r=0.923$ for the change in pAkt/Akt, $P<0.001)$. On Wes ${ }^{\circledR}$ we observed the distinct peaks around the expected molecular weights for these proteins with decreasing peak areas with serial dilutions of loading protein amount. Wes ${ }^{\circledR}$ and traditional western blot both had linear dynamic ranges for CD36, Erk1/2 and Akt. Due to differences in signal responsiveness for pAkt/Akt, we employed a calibrator sample and log transformation of data to allow proper comparisons. The Wes ${ }^{\circledR}$ approach required less sample than the traditional western blot and less technician/assay time, while achieving high sensitivity and good reproducibility. Capillary western technology $\left(\mathrm{Wes}^{\circledR}\right)$ provides a satisfactory alternative for analyses of human adipose tissue proteins.
\end{abstract}

\section{Introduction}

The traditional western blot is one of the most widely used analytical techniques to quantitate specific proteins in tissues and is considered the "gold standard". Recently, a novel capillary electrophoresis-based western blot technology has been developed by ProteinSimple, Inc. ${ }^{1}$. This capillary western blot assay incorporates sample separation performed in glass capillaries and immobilization of the proteins directly onto the capillary walls, followed by immuno-probing and chemiluminescent detection. This method has been validated for analyses of cell lysate proteins ${ }^{2}$, but whether it can be applied to adipose tissue extracts is unknown. Because the amount of published work on human adipose tissue proteins is considerably less than for cell or animal model systems, and because the lipid components of adipose tissue offer some challenges

\footnotetext{
Correspondence: Michael D. Jensen (jensen@mayo.edu)

'Department of Endocrinology, Changhai Hospital, Second Military Medical University, Shanghai, P.R. China

2Division of Endocrinology, Diabetes \& Metabolism, Endocrine Research Unit, Mayo Clinic College of Medicine, Rochester, MN, USA
}

that cell models don't, we wished to test whether the results from this new approach are as robust as those for traditional western blotting. For example, it may be important to understand whether differences in dynamic ranges and/or responses due to the much smaller amounts of sample with the capillary western blot assay require different analytical considerations. If the two approaches give similar results we and other investigators can be confident that data generated from Wes ${ }^{\circledast}$ is comparable to standard methods.

We elected to compare these two approaches to measure an adipose tissue protein amount, to measure the ratio of a phosphorylated protein to total protein, and to measure the changes in the phosphorylation state of an adipose tissue protein in response to an in vivo stimulus. Our rational was that if $\mathrm{Wes}^{\circledR}$ and western blotting were in good agreement for these three types of measurements we could have confidence in the technology for future applications.

The first protein we assessed was CD36, a transmembrane protein present in adipose tissue that facilitates cellular fatty 
acid uptake. The total amount of CD36 protein relates to in vivo adipose tissue fatty acid storage ${ }^{3}$. We also measured the ratio of phosphorylated Erk1/2 to total Erk1/2. Erk is an intracellular signaling pathway included in the mitogenactivated protein kinases (MAPKs) family. In adipose tissue, phosphorylated Erk1/2 is necessary to initiate the preadipocyte into the differentiation process ${ }^{4}$. Erk1 and Erk2 can be activated by insulin and by potentially by niacin acting via the beta-arrestin 1 pathway ${ }^{5}$. Finally, increases in the phosphorylation status of Akt in response to insulin are thought to regulate glucose uptake and inhibition of lipolysis in adipocytes. Because of our interest in adipose tissue insulin responses, we tested the ability of $\mathrm{Wes}^{\circledR}$ to measure the fold increase of $\mathrm{pAkt}^{\mathrm{S473}}$ (Akt phosphorylation on Ser473) in response to feeding compared to traditional western blotting. Our results indicate the two methods are in excellent agreement provided some aspects of sample loading and data handling are addressed.

\section{Materials and methods \\ Subjects}

The studies that were conducted to provide the samples were approved by the Mayo Clinic Institutional Review Board. Informed, written consent was obtained from all volunteers, who were healthy and receiving no medications.

For the examination of adipose tissue CD36, 7 volunteers were admitted to the Mayo Clinic Clinical Research Unit (CRU) the evening prior to the biopsies on two separate occasions. On one study day they underwent a 2-h euglycemic, hyperinsulinemic clamp with an insulin dose of 1.0 $\mathrm{mU} \mathrm{kg}{ }^{-1} \mathrm{~min}^{-1}$ and the other study day included a 2-h infusion of somatostatin $\left(200 \mu \mathrm{g} \mathrm{h}^{-1}\right)$ and epinephrine (13 $\mathrm{ng} \mathrm{kg}^{-1} \mathrm{~min}^{-1}$ ). At the end of the infusions on both days we performed abdominal and femoral adipose tissue biopsies.

The samples used to examine Erk $1 / 2$ were collected from 6 volunteers who had undergone a euglycemic, hyperinsulinemic $\left(0.5 \mu \mathrm{U} \mathrm{kg}^{-1} \mathrm{~min}^{-1}\right)$ clamp and six volunteers who had received two doses of extendedrelease niacin $(1 \mathrm{~g}$ at 0700 and another at $0800 \mathrm{~h}$ ). Abdominal subcutaneous fat biopsies were collected at $0830 \mathrm{~h}^{7}$ for both groups of volunteers.

For the examination of Akt, eight women (six non-obese) and four men (three non-obese) were admitted to CRU the evening prior to the study. At $0620 \mathrm{~h}$ the participants began consuming small portions of a liquid meal at 20-min intervals in order to increase plasma insulin concentrations as previously described ${ }^{8}$. Abdominal subcutaneous fat biopsies were collected at $0830 \mathrm{~h}$ (postprandial state). The participants remained in the CRU until the morning when they had repeat adipose tissue biopsies in the overnight fasted state?

We also made a calibrator sample from a pooled wholetissue extract of adipose tissue from surgical waste. The calibrator sample was used to test the protein detection, assay performance and dynamic range by both western blot and $\mathrm{Wes}^{\circledR}$.

\section{Reagents}

Wes-Rabbit (12-230 kDa) Master Kit (\# PS-MK04, \# PS-MK01) including anti-rabbit secondary antibody, antibody diluent, molecular weight ladder, streptavidinHRP, dithiothreitol (DTT), fluorescent master mix, luminol-S, peroxide, sample buffer and wash buffer were purchased from ProteinSimple (San Jose, CA, USA). This kit also provides capillary cartridge and pre-filled microplates. Antibodies used were specific for Erk1/2 (\#9102), pErk1/2 (\#9101), total Akt (\#4691), pAkt ${ }^{4473}$ (\#4060) (Cell Signaling) and CD36 (\#sc-9154, Santa Cruz). Kaleidoscope pre-stained standards (\#161-0324, Bio-Rad Laboratories, Inc.) were used as molecular weight marker in western blot.

\section{Adipose tissue extracts prepared for assays}

Adipose tissue from needle biopsies was washed with saline, frozen in liquid nitrogen, and stored at $-80^{\circ} \mathrm{C}$. For these assays, tissue was weighed and placed in a tube containing Omni ceramic homogenization beads (Omni International, Kennesaw, GA). Tissue was homogenized in SHBP (20 mM Tris-HCl, 1 mM EDTA, $255 \mathrm{mM}$ sucrose, PH 7.4, and anti-protease cocktail tablet from Roche), 1-4 $\mu \mathrm{L} \mathrm{mg}{ }^{-1}$ tissue at $4{ }^{\circ} \mathrm{C}$ using an Omni Bead Ruptor (speed $=2.10$, cycles $=2$, time $=15 \mathrm{~s}$, and delay $=10 \mathrm{~s}$ ). If the extract was prepared for testing pErk1/2 or pAkt, Halt phosphatase inhibitor cocktail (Thermo Scientific) was added to the homogenization buffer. The homogenate was centrifuged at $1000 \times g$ for $10 \mathrm{~min}$ at $4{ }^{\circ} \mathrm{C}$ and the subnatant (whole-tissue extract) below the lipid cake was aspirated into a new tube. Total protein concentrations of the whole-tissue extracts were measured using Pierce BCA Protein Quantitation kit (Thermo scientific) and the samples were stored at $-80^{\circ} \mathrm{C}$ until assayed.

\section{Western blot analyses}

Dilutions of whole-tissue extracts with NuPAGE ${ }^{\circledR}$ $4 \times$ LDS Sample Buffer were loaded on $10 \%$ NuPAGE $^{\circledR}$ Bis-Tris gels (Life Technologies). Proteins were separated by gel electrophoreses running on an Invitrogen Midi Gel system and transferred to polyvinylidene difluoride (PVDF) membranes on an Invitrogen semi-dry apparatus at $20 \mathrm{~V}$ for $1 \mathrm{~h}$. The membranes were blocked with $5 \%$ non-fat dry milk in TBS $(10 \mathrm{mM}$ Tris, $150 \mathrm{mM} \mathrm{NaCl}, \mathrm{pH}$ 7.4) for $1 \mathrm{~h}$ at room temperature. Primary antibodies (1:500 dilution for CD36, 1:1000 dilution for Erk1/2, pErk1/2, total Akt, and 1:2000 dilution for pAKT ${ }^{\text {S473 }}$ ) were incubated overnight at $4{ }^{\circ} \mathrm{C}$ in TBST (TBS with $0.1 \%$ Tween 20) with 5\% BSA. After four $5 \mathrm{~min}$ washes in TBST, the secondary antibody (Odyssey IRDye $800 \mathrm{CW}$ goat anti-rabbit-HRP, Li-Cor Biosciences) was 
incubated in a 1:10000-1:15000 dilution in TBST with $5 \%$ BSA for $1 \mathrm{~h}$ at room temperature followed by four 5 min washes in TBST. Detection of immuno-reactive bands was performed by image scan using a LI-COR Odyssey Imaging System (LI-COR Biosciences, Lincoln, NE).

\section{Capillary Wes analyses}

Capillary western analyses were performed using the ProteinSimple Wes ${ }^{\circledR}$ System. Samples (whole-tissue extracts) were diluted with 0.1 XSample Buffer. Then 4 parts of diluted sample were combined with 1 part $5 \times$ Fluorescent Master Mix (containing $5 \times$ sample buffer, $5 \times$ fluorescent standard, and $200 \mathrm{mM} \mathrm{DTT}$ ) and heated at $95^{\circ} \mathrm{C}$ for $5 \mathrm{~min}$. The Fluorescent Master Mix contains three fluorescent proteins that act as a "ruler" to normalize the distance for each capillary because the molecular weight ladder is only on the first capillary and each capillary is independent. After this denaturation step, the prepared samples, blocking reagent, primary antibodies (1:50 dilution for Erk1/2, pAkt ${ }^{\mathrm{S} 473}, 1: 100$ dilution for total Akt, and 1:150 dilution for CD36, pErk1/ 2 , the same antibodies as western blot), HRP-conjugated secondary antibodies and chemiluminescent substrate were dispensed into designated wells in an assay plate. A biotinylated ladder provided molecular weight standards for each assay.

After plate loading, the separation electrophoresis and immunodetection steps take place in the fully automated capillary system.

\section{Data and statistical analysis}

Intensities of bands in western blot and areas under peaks in Wes were detected to present for the relative amount of special proteins. A linear regression fit was used to determine the linear dynamic ranges of special proteins. Pearson correlation analysis was used to estimate the correlation of results from the western blot and Wes assay. Using the fold results in rank ordinal numbers, Spearman's rank correlation analysis was also used to measure the correlation of results from these two assays. A $p$-value of Correlation coefficient $<0.05$ was considered significant. Statistical tests were performed with SPSS version 19.0 for Windows (SPSS, Inc., Chicago, IL).

\section{Results}

\section{Operational principle of Wes ${ }^{\circledR}$}

Approximately $40 \mathrm{~nL}$ loading sample is injected into the capillary. Proteins are separated in capillaries as electrophoresis causes migration through a stacking and separation matrix ${ }^{10}$. The separated proteins are immobilized to the capillary wall via UV light cross-linking, following which the capillaries are washed with buffer to remove the gel matrix and are blocked with antibody diluent. The protein of interest is immuno-probed using a specific primary antibody (recommended dilution of 1:50-1:150), followed by an HRP-conjugated secondary antibody and the luminol-S/peroxide substrate. Data analysis is performed using the Compass Software $\left(\right.$ ProteinSimple $\left.{ }^{\circledast}\right)$. Molecular weight, the area under the peak, signal-to-noise ratio, peak height and peak width are reported for each named peak. The area under the peak represents the signal intensity of the immuno-detected protein.

\section{Qualitative analyses of CD36, Erk1/2 and Akt by western blot and Wes ${ }^{\circledR}$}

We used a whole-tissue extract of adipose tissue from surgical waste to test the protein detection by both western blot and $\mathrm{Wes}^{\oplus}$. Image results from traditional western blot provide main bands of CD36, total Erk1/2, pErk1/2 (phosphorylated Erk1/2), total Akt and pAkt ${ }^{\mathrm{S} 73}$ (phosphorylated Akt on Ser473) as we expect. On Wes ${ }^{\circledR}$ we observed distinct peaks around the expected molecular weights for these proteins $(120 \mathrm{kDa}$ for CD36, two peaks of $42 \mathrm{kDa}$ and $44 \mathrm{kDa}$ for Erk1/2, $60 \mathrm{kDa}$ for Akt), with decreasing peak areas with serial dilutions of loading samples (Fig. 1, right panel). Wes ${ }^{\circledast}$ also could provide a gel-like image view, showing the decreasing intensities of bands with serial dilutions of loading samples (Fig. 1, left panel).

\section{Comparison of dynamic range}

As shown in Fig. 2, both $\mathrm{Wes}^{\circledR}$ and traditional western blot have linear dynamic ranges for CD36, Erk1/2 and Akt. However, Wes ${ }^{\circledR}$ uses much less sample relative to traditional western blot. The linear relationship between signal intensity and total protein for the western blot was acceptable in the ranges of 5.0-20.0 $\mu$ g for measuring CD36, 2.6-42.2 $\mu \mathrm{g}$ for measuring Akt and $11.7-93.9 \mu \mathrm{g}$ for measuring Erk1/2. For the $\mathrm{Wes}^{\circledR}$ the linear relationship for signal intensity was acceptable for total protein amounts of 4.0-64.0 ng for CD36, $0.2-3.0 \mathrm{ng}$ for Akt and 2.7-42.5 ng for Erk1/2.

\section{Assay performance}

We used a calibrator sample obtained from a pooled whole adipose tissue extract to test the reproducibility of $\mathrm{Wes}^{\circledast}$. Using high, medium and low dilution of a calibrator sample on every run over $>4$ months, we found the inter-assay CV's for CD36 and for the ratio of pErk1/ 2 to total Erk1/2 were both $10 \%$ and for the ratio of $\mathrm{pAkt}^{\mathrm{S} 473}$ to total Akt was $15 \%$. Using the calibrator sample 10 times on a single run we found the intra-assay $\mathrm{CV}$ of the ratio of $\mathrm{pAkt}^{\mathrm{S} 473}$ to total Akt was $14 \%$ for $\mathrm{Wes}^{\circledR}$. For reference purposes we also tested the assay performance of traditional western blotting for the ratio 


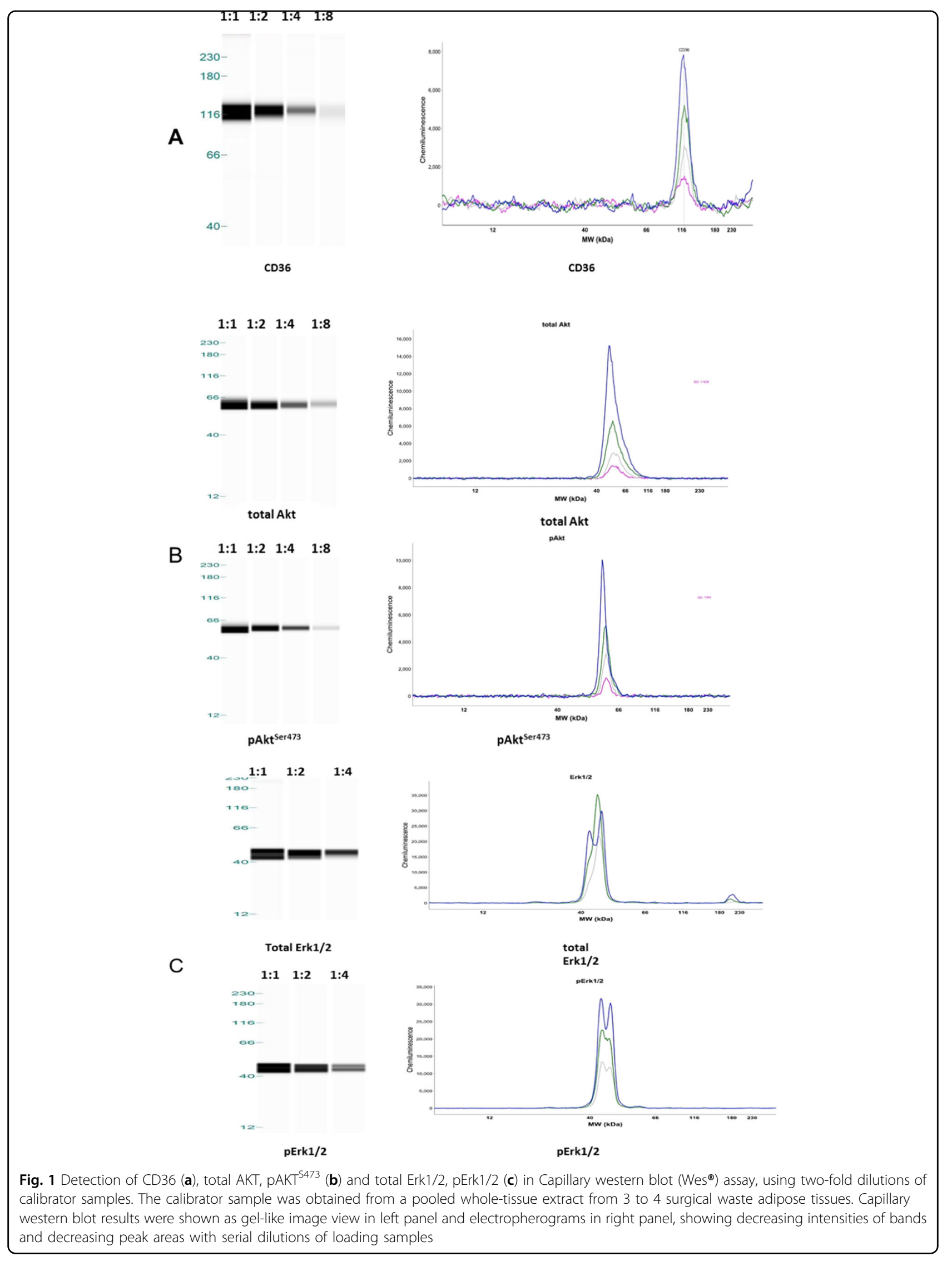




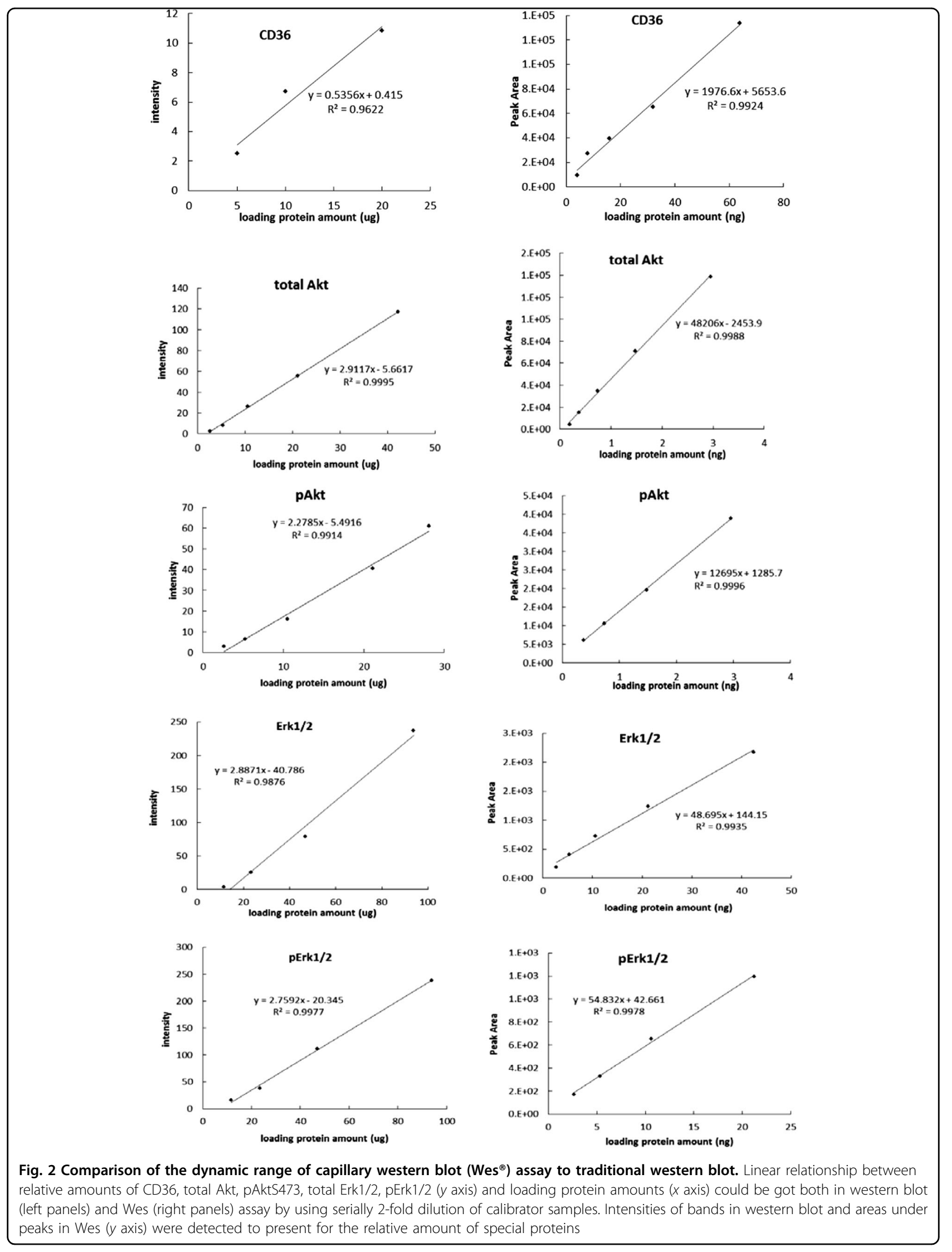




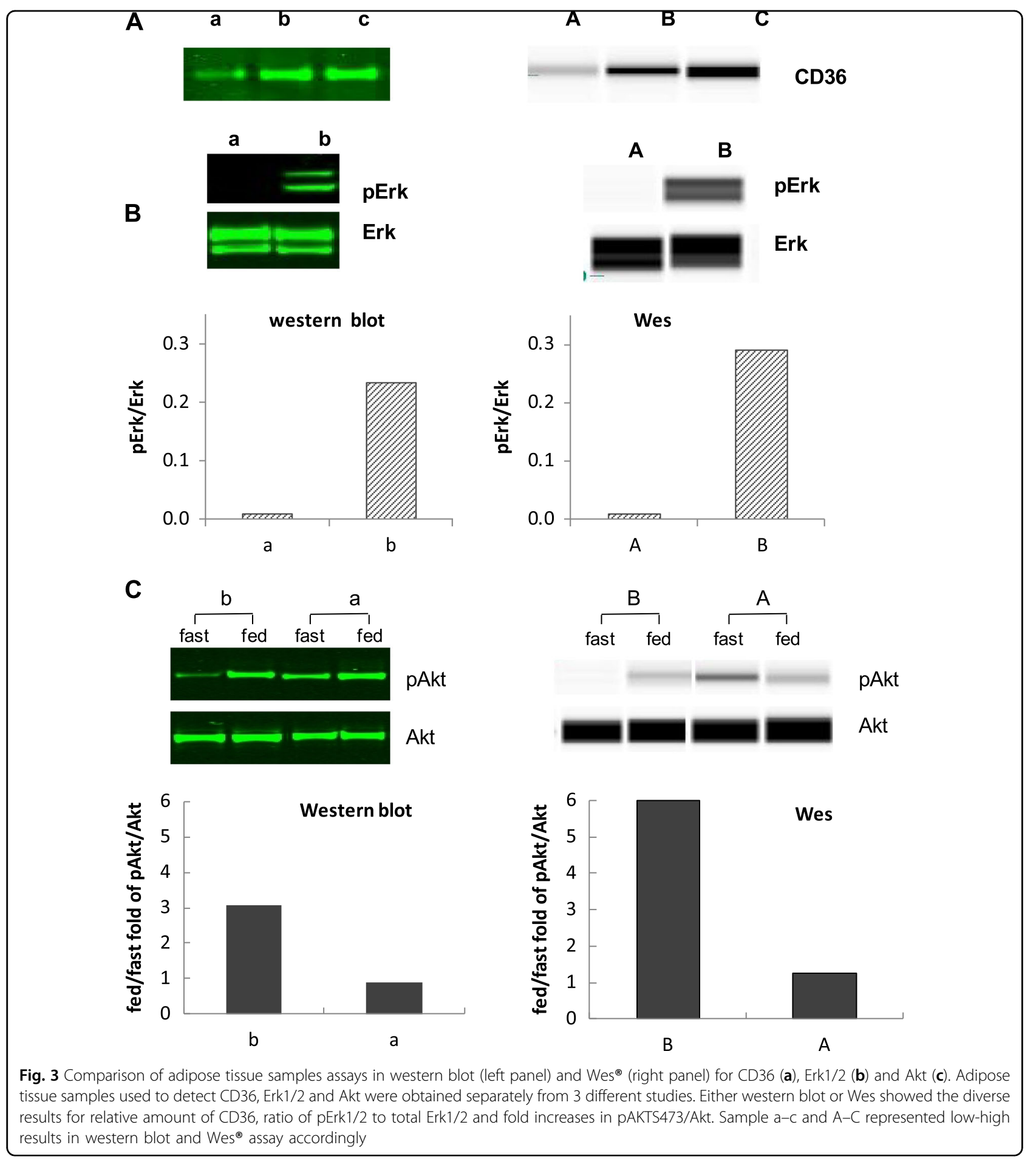

of pAkt ${ }^{\mathrm{S} 473}$ to total Akt and found it to be comparable $(18 \%)$.

\section{Comparison of CD36 between western blot and Wes ${ }^{\circledR}$ results}

We analyzed paired 17 adipose samples (abdomen or thigh) from 7 different subjects obtained under hyperinsulinemic or hypoinsulinemic/epinephrine conditions to determine the agreement between $\mathrm{Wes}^{\circledR}$ and western in quantitating CD36 protein levels. Figure 3a depicts the examples of results from samples run on traditional western blot (left side) and $\mathrm{Wes}^{\circledR}$ (right side). The results from these two methods were highly correlated ( $r=0.932, P<0.001$, Fig. 4 , top panel). Using 
the fold results in rank ordinal numbers, the Spearman rank correlation coefficient remained significant $(r=0.931, P<0.001)$.

\section{Comparison of Erk1/2 between western blot and Wes ${ }^{\circledR}$ results}

We analyzed paired adipose samples from 14 different subjects obtained under hyperinsulinemic or niacin exposed conditions to determine the agreement between $\mathrm{Wes}^{\circledast}$ and western in quantitating the ratio of $\mathrm{pErk} 1 / 2$ to Erk $1 / 2$. Figure $3 b$ depicts the results from 2 of these samples run on traditional western blot (left side) and Wes $^{\circledR}$ (right side) for pErk1/2 to Erk1/2 Wes ${ }^{\circledast}$. The results from these two methods were highly correlated ( $r=0.905, P<0.001$, Fig. 4, middle panel). Using the fold results and rank ordinal numbers, the Spearman rank correlation coefficient remained significant $(r=0.912, P<0.001)$.

\section{Approaches to allow between method comparisons}

One of our goals was to determine whether changes in Akt phosphorylation are comparable using $\mathrm{Wes}^{\circledR}$ and traditional western blotting. In the course of testing four different surgical waste adipose tissue samples using traditional western blot approaches we found different responses between the loading protein amount and the signal intensity for total Akt (Fig. 5a). The implication of this observation is that between-sample differences in adipose tissue Akt content are not linearly related, even if identical amounts of protein are loaded. Of interest, the lines describing the relationships for these four samples were virtually parallel after log-transforming both $x$ axis (loading protein amount) and $y$ axis (intensity) data (Fig. 5b). We also found that the relationships between protein amount and signal intensity for total Akt and $\mathrm{pAkt}^{\mathrm{S} 473}$ in our calibrator sample were different when plotted using unadjusted data for both traditional western blotting (Fig. 5c) and the Wes ${ }^{\circledast}$ (Fig. 5e). These differences in the slopes of the responses would make it impossible to compare changes in the ratio of pAkt to total Akt between traditional western blotting and Wes ${ }^{\circledR}$. We found that the relationships became virtually parallel for both western blot (Fig. 5d) and $\mathrm{Wes}^{\circledR}$ (Fig. 5f) if we $\log$ transformed the protein and signal intensity data, allowing us to compare the changes in pAkt:Akt between traditional western blotting and $\mathrm{Wes}^{\circledR}$.

\section{Comparison of Akt between western blot and Wes ${ }^{\circledast}$ results}

We analyzed paired adipose samples from 12 different subjects obtained in the fasted and fed states to determine the agreement between $\mathrm{Wes}^{\circledR}$ and western in quantitating the fold increase in pAkt/Akt. Figure 3, panel C depicts the diverse fold increases in $\mathrm{pAKT}{ }^{\mathrm{S} 473}$ /Akt between the fasting and fed state for two subjects using both

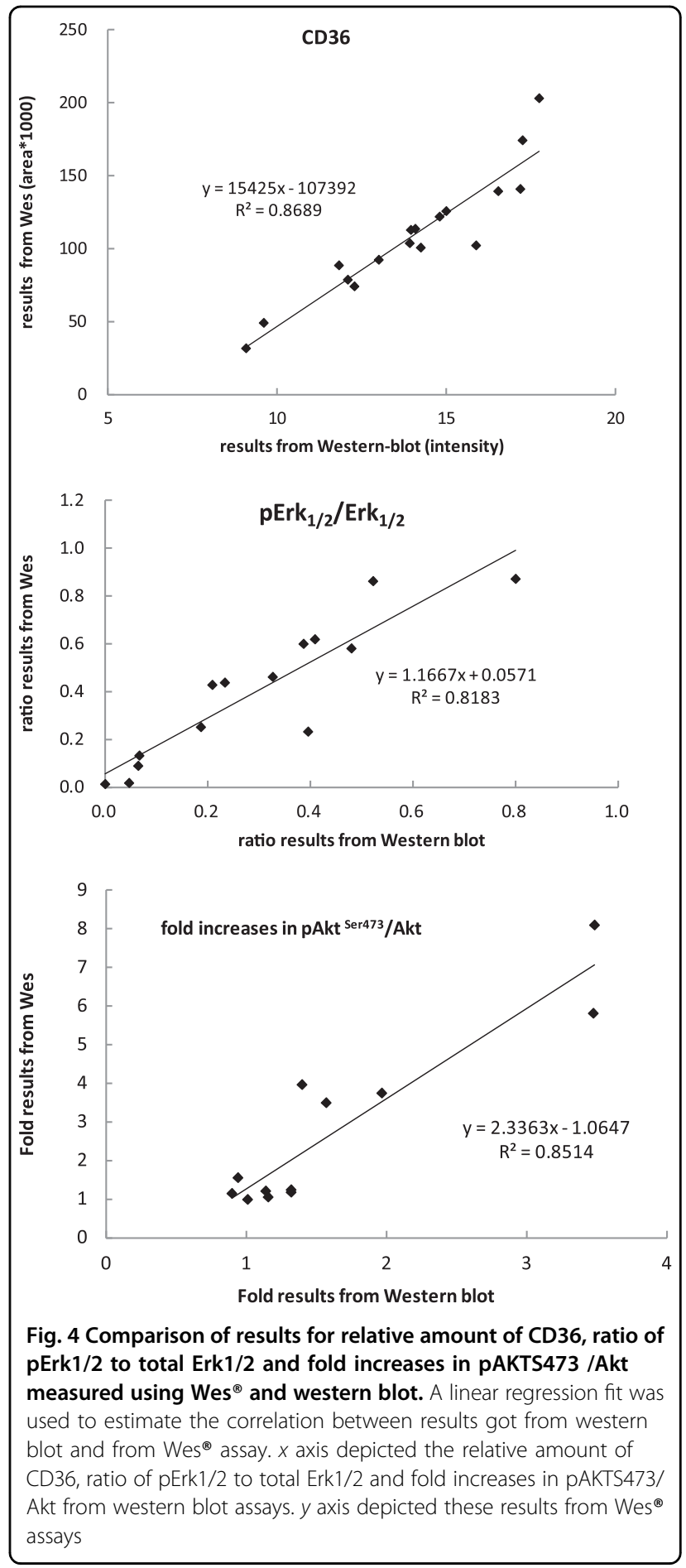

traditional western blot (upper) and Wes ${ }^{\circledR}$ (lower) methods. The results from the two methods were highly correlated $(\mathrm{r}=0.923, \mathrm{P}<0.001$, Fig. 4 , bottom panel). Using the fold results a rank ordinal numbers, the Spearman rank correlation coefficient remained significant $(r=0.797, P=0.002)$. 
A.

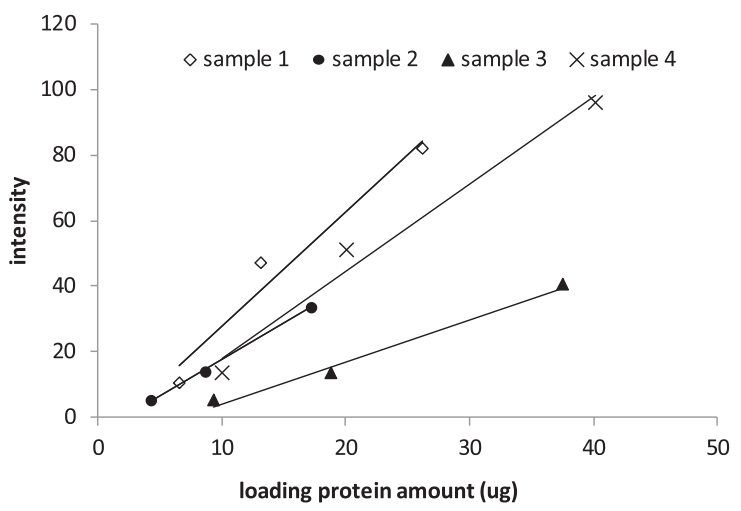

C.

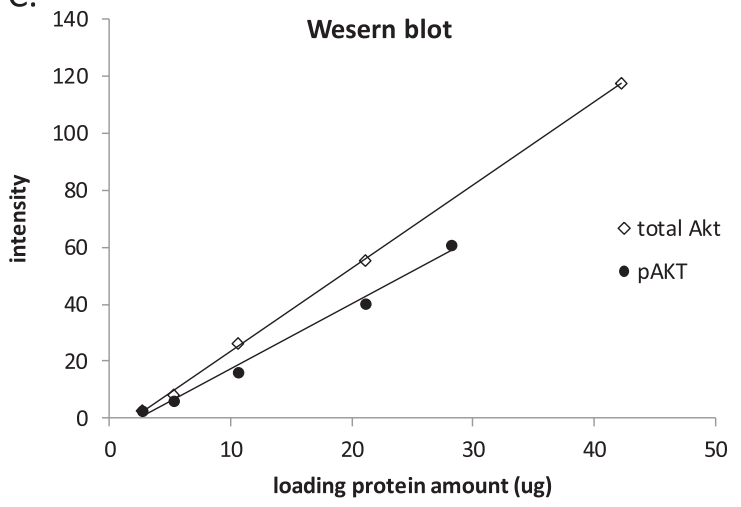

E.

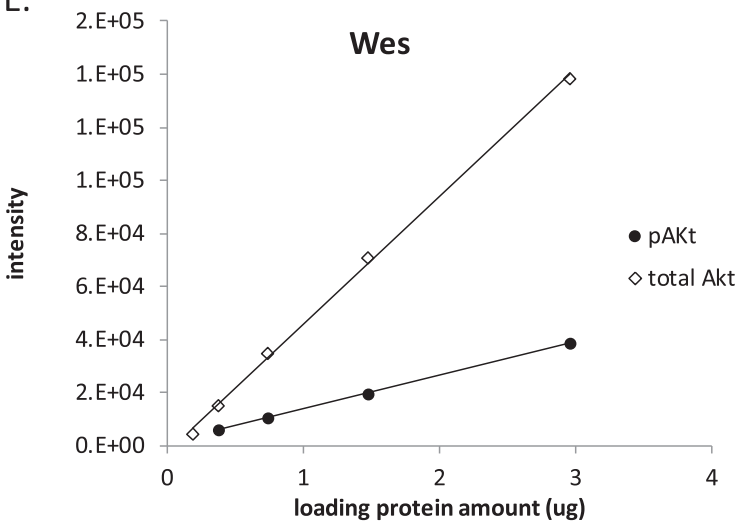

B.

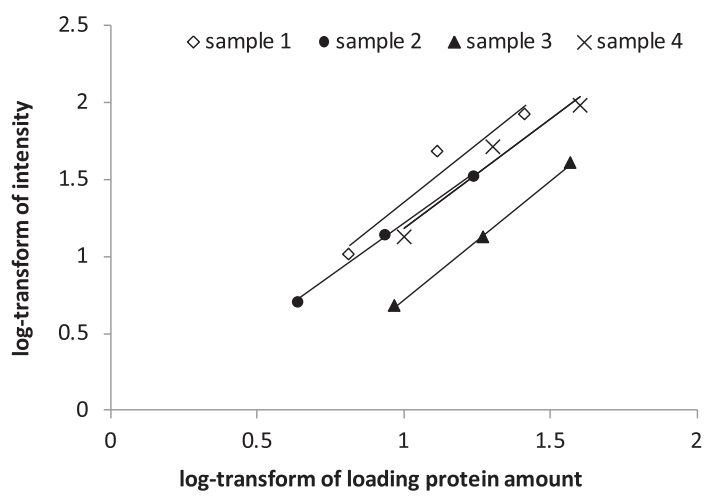

D.

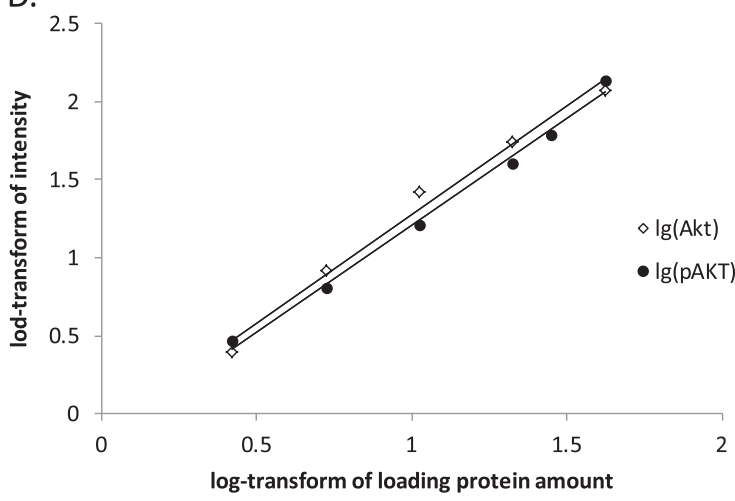

F.

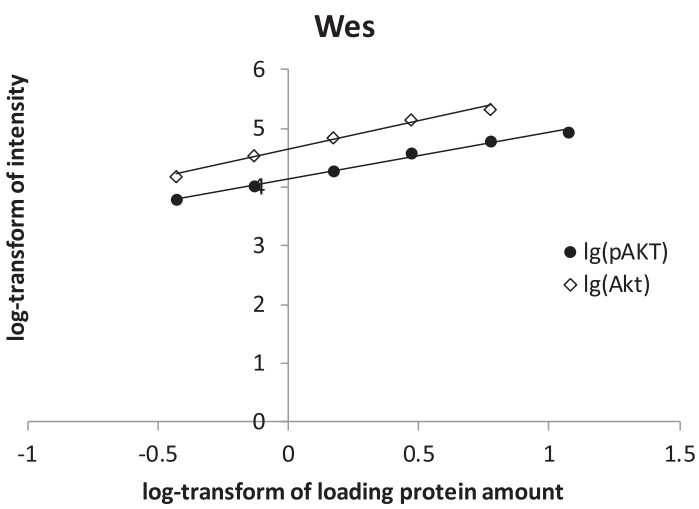

Fig. 5 The regression lines of four adipose tissue samples for tested total Akt and QC sample for total Akt and pAktS473 before and after log-transform in western blot and Wes ${ }^{\circledR}$ analyses. a The regression lines of different adipose tissue extracts are not parallel. After log-transforming both $x$ axis (loading protein amount) and $y$ axis (intensity), the regression lines of these four samples become parallel (b). $\mathbf{c}$, e The regression lines of QC sample for total Akt and pAktS473 tested by western blot and Wes ${ }^{\circledR}$. $\mathbf{d}, \mathbf{f}$ The regression lines of QC sample for total Akt and pAktS473 after logtransform in western blot and Wes ${ }^{\circledast}$

\section{Discussion}

Because capillary western approaches offer investigators the ability to measure multiple proteins and require much smaller amounts of tissue, we wished to test whether this method compares favorably to traditional western blotting for human adipose tissue proteins. We found that the ProteinSimple $\mathrm{Wes}^{\circledast}$, a more sensitive and automated approach for protein detection and 
characterization, provided comparable results to traditional western blotting. We also encountered some challenges that relate to nuances of signal responsiveness of the two methods. The capillary western approach for measuring protein content (CD36), the phosphorylation state of a protein (pErk1/2:Erk1/2), and even the change in pAkt/Akt in response to meal ingestion agreed well between the two methods. We conclude that this capillary western method of measuring proteins in adipose tissue is a valid approach that requires less tissue and is less labor intensive.

Previous studies described the application of such capillary western blot system for vaccine development ${ }^{11}$, biopharmaceutical fusion-Fc protein evaluation $^{12}$ and signal pathway modulation in cell samples ${ }^{13}$. Other reported applications of this capillary western system include specific biomarkers for diabetes and cancer research ${ }^{14}$. Because we found no publications of its use for protein analyses in human adipose tissue we sought to understand whether capillary western approaches can compete with traditional western blotting.

Traditional western blot requires many time consuming steps, including sample loading, gel electrophoresis, transfer, blocking, primary and secondary antibody incubation and detection ${ }^{15}$. Because all of these steps are manual it is sometimes challenging to become proficient and maintain consistent results. However for $\mathrm{Wes}^{\circledR}$, the separation electrophoresis and immune detection steps take place fully automated in the glass capillaries after plate loading ${ }^{16}$. We found that the reproducibility of automated capillary western approach is equal to or better than traditional western blotting, perhaps because it reduces the chance for operator error. It also requires less time for sample preparation and plate loading $(\sim 2 \mathrm{~h})$ and less total run time $(\sim 3 \mathrm{~h})$. Although the supply expenses are greater for capillary western methods, the time savings and greater sensitivity (requiring less sample) are offsetting advantages.

Of the three proteins we selected for comparison, measuring the fold increase in $\mathrm{pAkt}^{\mathrm{S} 473} / \mathrm{Akt}$ in response to feeding was most challenging. To measure the ratio of pErk $1 / 2$ to Erk1/2, we could load the same protein amount for Erk1/2 and pErk1/2. In contrast, to be able to observe the fold increase of $\mathrm{pAkt}^{\mathrm{S} 473}$ we were required to load different amounts of protein for the fasting and fed samples. This was because fasted $\mathrm{pAkt}^{8473}$ was very low in many samples. In cases where the fasting $\mathrm{pAkt}^{\mathrm{S} 473} / \mathrm{Akt}$ was at or near zero, we could not calculate the fold increase. However, loading more protein to detect $\mathrm{pAkt}^{\mathrm{S} 473}$ was not a viable approach because the intensity of total Akt would exceed the top of linear dynamic range, especially in $\mathrm{Wes}^{\circledR}$. To address the need to load different protein amounts for total Akt and $\mathrm{pAkt}^{\mathrm{S} 473}$ assays we used a calibrator sample as a quality control $(\mathrm{QC})$, allowing us to calculate the relative amount of signal to the QC and thereby calculate the ratio of $\mathrm{pAkt}^{\mathrm{S} 733}$ to total Akt. Furthermore, by logtransforming both the loading protein amount and the signal intensity we could achieve parallel lines describing the relationships for total Akt and $\mathrm{pAkt}^{\mathrm{S} 473}$ in both western blot and Wes ${ }^{\circledR}$ assays by testing the calibrator sample. This allowed us to compare fold increase in using the two approaches, which was not possible absent this solution.

In summary, our results indicate that capillary western approaches using ProteinSimple $\mathrm{Wes}^{\oplus}$ provides an attractive alternative for protein analyses of human adipose tissue. We found the approach to be time-saving, with high sensitivity, good quantitation and reproducibility.

\section{Acknowledgements}

We thank Deborah Harteneck for assistance with the preparation of adipose tissue extracts for these analyses.

\section{Funding}

Grant Support: NCRR 1UL1 RR024150, NIH grants DK45343 and DK40484. Dr. Lu was sponsored by Natural Science Foundation of Shanghai (15ZR1413100).

\section{Conflict of interest}

The authors declare that they have no conflict of interest.

Received: 31 January 2017 Revised: 19 June 2017 Accepted: 7 August 2017 Published online: 25 April 2018

\section{References}

1. Nguyen U., Squaglia N., Boge A., Fung P. A. The Simple western[trade]: a gelfree, blot-free, hands-free western blotting reinvention. Nat. Methods 2011; 8 http://dx.doi.org/10.1038/nmeth.f.353

2. Chen, J. Q. et al. Absolute quantitation of endogenous proteins with precision and accuracy using a capillary western system. Anal. Biochem. 442, 97-103 (2013).

3. Hames, K. C., Vella, A., Kemp, B. J. \& Jensen, M. D. Free fatty acid uptake in humans with CD36 deficiency. Diabetes 63, 3606-3614 (2014).

4. Bost, F., Aouadi, M., Caron, L. \& Binetruy, B. The role of MAPKs in adipocyte differentiation and obesity. Biochimie 87, 51-56 (2005).

5. Walters, R. W. et al. beta-Arrestin1 mediates nicotinic acid-induced flushing, but not its antilipolytic effect, in mice. J. Clin. Invest. 119, 1312-1321 (2009).

6. Siddle, K. Signalling by insulin and IGF receptors: supporting acts and new players. J. Mol. Endocrinol. 47, R1-R10 (2011).

7. Ali, A. H., Mundi, M., Koutsari, C., Bernlohr, D. A. \& Jensen, M. D. Adipose tissue free fatty acid storage in vivo: effects of insulin versus niacin as a control for suppression of lipolysis. Diabetes 64, 2828-2835 (2015).

8. Muthusamy, K. et al. Effect of insulin infusion on spillover of meal-derived fatty acids. J. Clin. Endocrinol. Metab. 97, 4201-4205 (2012).

9. Koutsari, C., Mundi, M. S., Ali, A. H. \& Jensen, M. D. Storage rates of circulating free fatty acid into adipose tissue during eating or walking in humans. Diabetes 61, 329-338 (2012).

10. Shihabi, Z. K. Stacking in capillary zone electrophoresis. J. Chromatogr. A $\mathbf{9 0 2}$ 107-117 (2000).

11. Rustandi, R. R. et al. Qualitative and quantitative evaluation of Simon, a new CE-based automated western blot system as applied to vaccine development. Electrophoresis 33, 2790-2797 (2012). 
12. Xu, D., Mane, S. \& Sosic, Z. Characterization of a biopharmaceutical protein and evaluation of its purification process using automated capillary western blot. Electrophoresis 36, 363-370 (2015)

13. Klein, B. Y., Tamir, H., Hirschberg, D. L., Glickstein, S. B. \& Welch, M. G. Oxytocin modulates mTORC1 pathway in the gut. Biochem. Biophys. Res. Commun. 432, 466-471 (2013).
14. Liu, S.-B. et al. The application of a novel nanovolume capillary electrophoresisbased protein analysis system in personalized \& translational medicine research. J. Bioanal. Biomed. S3, 004 (2013).

15. Kurien, B. T. \& Scofield, R. H. western blotting. Methods 38, 283-393 (2006).

16. O'Neill, R. A. et al. Isoelectric focusing technology quantifies protein signaling in 25 cells. Proc. Natl Acad. Sci. USA 103, 16153-16158 (2006). 